\title{
Shedding New Light on Thermionic Electron Emission of Fullerenes
}

\author{
G. von Helden, ${ }^{1}$ I. Holleman, ${ }^{1}$ A. J. A. van Roij, ${ }^{1}$ G. M. H. Knippels, ${ }^{2}$ A. F. G. van der Meer, ${ }^{2}$ and G. Meijer ${ }^{1}$ \\ ${ }^{1}$ Department of Molecular and Laser Physics, University of Nijmegen, Toernooiveld, NL-6525 ED Nijmegen, The Netherlands \\ ${ }^{2}$ FOM-Institute for Plasma Physics Rijnhuizen, Edisonbaan 14, NL-3430 BE Nieuwegein, The Netherlands
}

(Received 25 February 1998)

\begin{abstract}
Tunable pulsed infrared (IR) radiation from a free electron laser is used to selectively excite fullerenes via their vibrational modes to very high internal energies. After absorbing several hundred IR photons, the molecules can autoionize. Ion creation is unexpectedly slow, occurring at times beyond $0.1 \mathrm{~ms}$ after laser excitation, but is found to be significantly faster for electronically preexcited molecules. It is concluded that reaching the first electronically excited state is a high hurdle on the way from hot molecules to ions. [S0031-9007(98)06980-4]
\end{abstract}

PACS numbers: $36.40 . V z, 33.20 . E a, 33.80 . R v$

For a long time, the microscopic equivalent of the thermal emission of electrons from a heated surface, the thermionic emission of electrons from neutral hot molecules, was not observed. For thermionic emission to occur, a large amount of vibrational energy has to be converted into electronic energy and the probability and mechanism of such a process is not a priori clear. During the last decade, however, delayed electron emission has been observed for metal clusters [1-3] as well as for fullerenes [4-12]. Although for $\mathrm{C}_{60}$ the influence of multiple excited electronic states is still being discussed [8], most experiments indicate that this delayed electron emission cannot be attributed to a direct (electronic) ionization process, and it is considered to be a signature for the thermal emission of electrons. Further evidence for a thermal emission mechanism comes from theoretical models [13-17] that can describe the experimental observations [6,9]. In all these experiments the clusters (molecules) are excited using pulsed visible (VIS) or ultraviolet (UV) lasers, thus initially preparing electronically excited states. These states can relax to high vibrationally excited levels of the electronic ground state surface from where the thermal ionization is assumed to occur.

More direct insight in the process of thermionic electron emission can be obtained when the molecules are directly excited vibrationally by using infrared (IR) lasers [18-20]. We here report on the dynamics of the electron emission from gas-phase fullerenes that are resonantly excited on strong IR active modes using a tunable free electron laser (FEL). By absorbing several hundred IR photons per molecule from the train of high power subpicosecond pulses from the FEL, the molecules are efficiently heated and thermionic electron emission can occur. The time evolution of the production of the ions following IR excitation is recorded both for "normal" and for electronically preexcited $\mathrm{C}_{60}$. The results obtained are rather different from the results obtained when UV/VIS lasers are used and shed new light on the mechanism of thermal ionization.

Experiments are performed at the free electron laser for infrared experiments (FELIX) $[21,22]$ in Nieuwegein, The
Netherlands. This laser produces IR radiation that is continuously tunable over the $100-2000 \mathrm{~cm}^{-1}$ range. The light output consists of macropulses of about $4 \mu$ s duration containing up to $50 \mathrm{~mJ}$ of energy. Each macropulse consists of a train of micropulses that are approximately 0.3 ps long and $1 \mathrm{~ns}$ apart. The macropulse repetition rate is $10 \mathrm{~Hz}$. A scheme of the experimental setup, as constructed inside a compact turbopumped vacuum chamber, is depicted in Fig. 1. A quartz oven, filled with high purity $\mathrm{C}_{60}$ powder and kept at a temperature of $700-800 \mathrm{~K}$, is used to generate an effusive beam of fullerenes. The IR laser beam enters the apparatus slightly above the quartz oven and is focused by a spherical mirror with a $7.5 \mathrm{~cm}$ focal length in the center between the plates of a timeof-flight (TOF) mass spectrometer. A second mirror with a focal length of $3.75 \mathrm{~cm}$ is used as retroreflector, effectively doubling the micropulse repetition rate in the focus to $2 \mathrm{GHz}$. The focusing arm of the IR cavity makes an angle of $23^{\circ}$ with the axis of the molecular beam, thereby

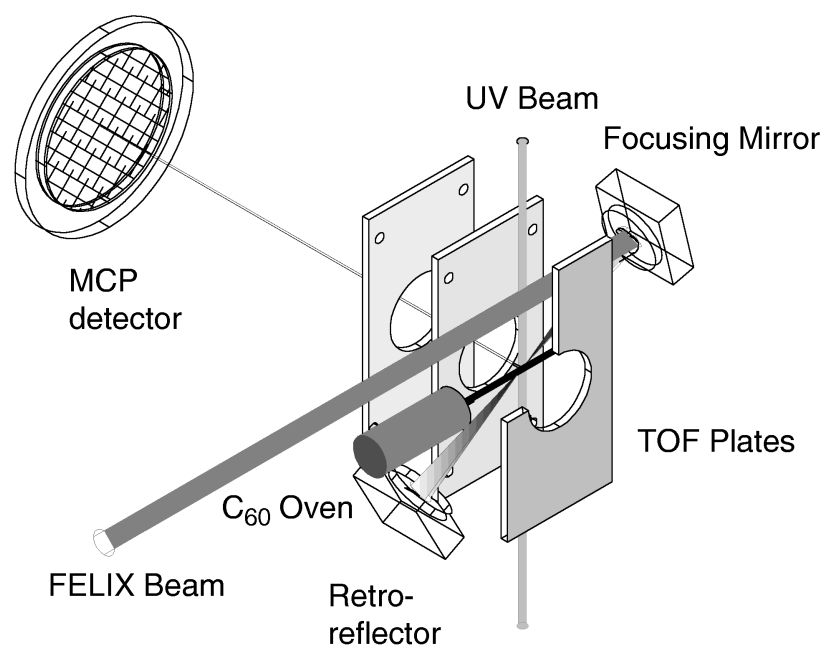

FIG. 1. Scheme of the experimental setup. An effusive beam of fullerenes originates from a quartz oven and is crossed by laser beams in the center between two electrodes. Laser produced ions are accelerated and detected on a multichannel plate (MCP) detector. 
lengthening the interaction time of fullerenes with the focused IR beam compared to a perpendicular geometry. The TOF setup consists of three rectangular stainless steel electrodes that are placed $1.5 \mathrm{~cm}$ apart. The center of the plates consists of a circular grid pattern of $2.5 \mathrm{~cm}$ diameter with an optical transmission of $85 \%$ to allow ions to pass while keeping the electric fields homogeneous. Ions are detected on a double multichannel plate (MCP) detector at a distance of $45 \mathrm{~cm}$ from the last TOF electrode. The experimental geometry allows for the coupling in of an extra laser to perform double-resonance experiments. In the two-color experiments we use the (unfocused) quadrupled output from a Nd:YAG laser $(266 \mathrm{~nm} ; 50-70$ ps pulse duration) to electronically excite the fullerenes prior to the IR excitation with FELIX. In those experiments, the output power of the Nd:YAG laser is adjusted such that no ionization is observed from the UV light alone. To record IR resonance enhanced multiphoton ionization (IR-REMPI) spectra of $\mathrm{C}_{60}$, the TOF electrodes are pulsed to high voltage some $30 \mu \mathrm{s}$ after the FELIX pulse, and the total number of $\mathrm{C}_{60}^{+}$ions is recorded as a function of IR laser wavelength. To record the time evolution of the production of ions, the total ion signal is measured as a function of time with the voltage on the TOF electrodes being constantly on (electric field of $600 \mathrm{~V} / \mathrm{cm}$ in the extraction region) and the IR laser being fixed in frequency on a resonance. From the measured arrival time distribution of the ions at the detector, the flight time is subtracted to obtain the "time-of-birth" (TOB) distribution. These TOB distributions are the increment in total ion signal as a function of time; i.e., they correspond to the rate of ion production (multiplied by the time constant of the detection system).

In Fig. 2, TOB distributions resulting from the excitation of $\mathrm{C}_{60}$ on the IR active mode at $19.2 \mu \mathrm{m}\left(520 \mathrm{~cm}^{-1}\right.$; see also Fig. 3) are shown for three different macropulse energies. The envelope of the FELIX pulse is shown in the lower part of the figure. At long times, the chances for an ion to be detected diminish, since the neutral precursors escape from the detection region between the TOF electrodes. Using the experimental geometry and a Maxwellian $\mathrm{C}_{60}$ velocity distribution, the experimental detection efficiency curve shown in the lower part of the figure is calculated. Several important points can immediately be recognized from Fig. 2. First, at all excitation fluences, the TOB distributions extend to very long times. Taking the experimental detection efficiency into account, it is evident that ions are produced at $150 \mu$ s and beyond. This corresponds to a $(1 / e)$ autoionization time scale that is significantly larger than previously observed [4-6,12]. Second, and even more surprising, the two TOB distributions taken at low fluence are clearly peaked after the end of the FELIX pulse and appear to consist of at least two components. When increasing the laser fluence, the fastest of these components gains dramatically in intensity relative to the slow component, and both components are seen to have their maxima shifted to shorter times. At the highest

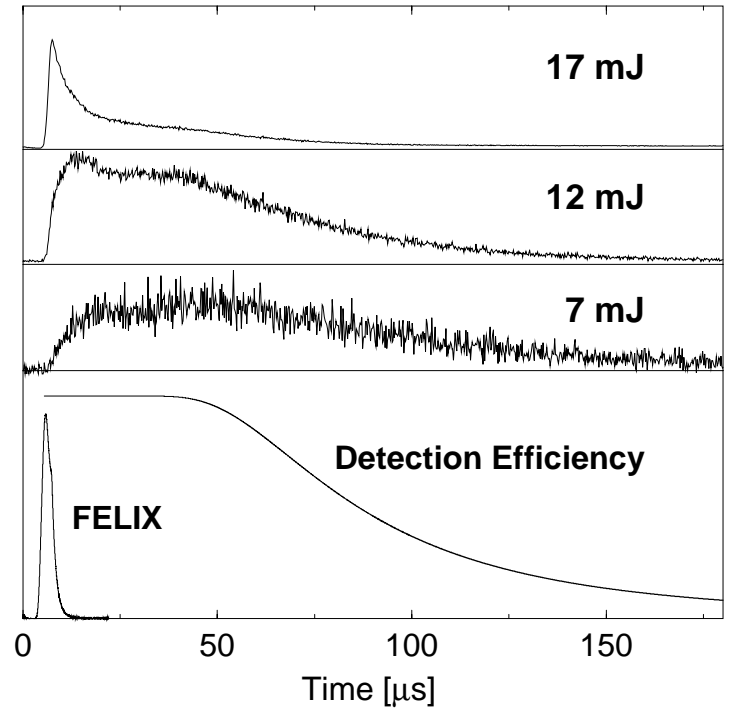

FIG. 2. Time-of-birth (TOB) distributions of $\mathrm{C}_{60}^{+}$ions at three different macropulse energies. The envelope of the FELIX micropulses is shown in the lower part together with the time dependence of the experimental detection efficiency.

laser fluence, the TOB distribution peaks during the FELIX pulse but the slow component can still be recognized as a shoulder in the otherwise fast decaying transient. It is clear that none of the TOB distributions can be described by either a single exponentially decaying function [4-6] or a power function of time [17], functions that are successfully used to describe the time dependence of autoionization when the molecules are excited with UV/VIS lasers.

The observation that the TOB distributions are peaked after the FELIX pulse cannot be explained by a model that assumes a population of excited molecules that undergoes a first-order reaction. In such a model, the rate of ion production is directly proportional to the excited state population and an exponentially decaying function

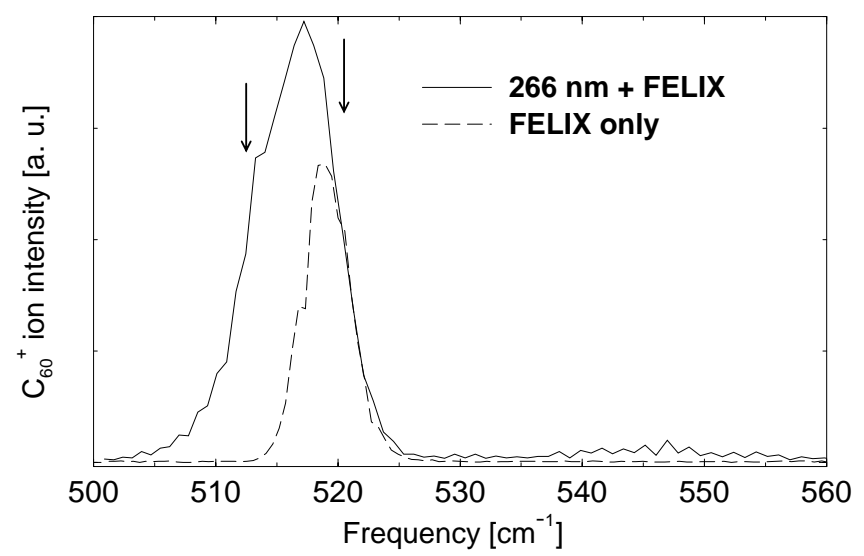

FIG. 3. IR-REMPI spectrum obtained after preexciting $\mathrm{C}_{60}$ with $266 \mathrm{~nm}$ radiation to its triplet state (solid line) compared to the IR-REMPI spectrum of $\mathrm{C}_{60}$ as obtained with the IR laser only (dashed line). The arrows indicate the IR laser frequencies that are used to record the TOB distributions shown in Fig. 4. 
results. Even when such exponentially decaying functions are convoluted with an internal energy distribution, a monotonically decaying function will result. The simplest unimolecular model that can give rise to a distribution that peaks at a later time must include an intermediate step:

$$
\mathrm{C}_{60}+n h \nu \longrightarrow \mathrm{C}_{60}^{*} \stackrel{k_{1}}{\longrightarrow} \mathrm{C}_{60}^{\#} \stackrel{k_{2}}{\longrightarrow} \mathrm{C}_{60}^{+}+e^{-},
$$

in which $\mathrm{C}_{60}^{*}$ stands for the laser excited $\mathrm{C}_{60}$ molecules that can convert with the rate constant $k_{1}$ to the intermediate state $\mathrm{C}_{60}^{\#}$. This intermediate state can then undergo autoionization with a rate constant $k_{2}$. Such a model gives rise to TOB distributions of the functional form

$$
\operatorname{TOB}(t) \propto\left(e^{-k_{1} t}-e^{-k_{2} t}\right) /\left(k_{2}-k_{1}\right) .
$$

This function starts at zero at $t=0$, rises to a maximum at $t=\left(k_{2}-k_{1}\right)^{-1} \ln \left(k_{2} / k_{1}\right)$, and then decays. Similar functions are obtained when more than one intermediate step is included in reaction scheme (1).

In order to investigate the possible role of electronically excited states as intermediate states, we performed a double-resonance experiment, in which $\mathrm{C}_{60}$ first absorbs one $266 \mathrm{~nm}(4.7 \mathrm{eV})$ photon. After excitation of $\mathrm{C}_{60}$ with the UV photon, the excited molecules will undergo rapid intersystem crossing to low lying triplet states [23] which are known to have a long lifetime at low internal energies [24]. Five microseconds after the UV excitation, FELIX is fired. The IR photons will thus probe $\mathrm{C}_{60}$ in its triplet state and IR-REMPI spectra as well as TOB distributions of triplet excited $\mathrm{C}_{60}$ can be obtained.

The solid line in Fig. 3 shows the IR-REMPI spectrum of $\mathrm{C}_{60}$ in the 500 to $560 \mathrm{~cm}^{-1}$ region after preexcitation with the UV laser. The dashed line is the normal IRREMPI spectrum of (singlet) $\mathrm{C}_{60}$ [20], obtained when exciting with FELIX only, using the same IR power and fluence as in the double resonance experiment. In order to obtain the true triplet IR-REMPI spectrum, the dashed spectrum has to be subtracted from the solid spectrum as the UV laser will excite only a fraction of the molecules in the interaction volume. It is clear from comparing the two spectra that the ion signal on the red side of the peak results solely from triplet excited $\mathrm{C}_{60}$, and it appears that the IR resonance of triplet $\mathrm{C}_{60}$ is shifted to lower frequency by about $5 \mathrm{~cm}^{-1}$ relative to the corresponding IR resonance in ground state $\mathrm{C}_{60}$.

In Fig. 4 two different TOB distributions are shown. The upper curve is taken in the two-color experiment with FELIX at $512 \mathrm{~cm}^{-1}$. All of the signal has to result from the IR excitation of triplet $\mathrm{C}_{60}$, as ground state $\mathrm{C}_{60}$ does not give rise to an IR-REMPI signal at this FELIX frequency. The lower curve is taken at $520 \mathrm{~cm}^{-1}$, without UV preexcitation. In both experiments, the IR laser power and fluence is kept the same. Both curves consist of a fast and a slow component, and the TOB distribution without UV preexcitation appears to be intermediate between the upper two TOB distributions in Fig. 2. Each of the two

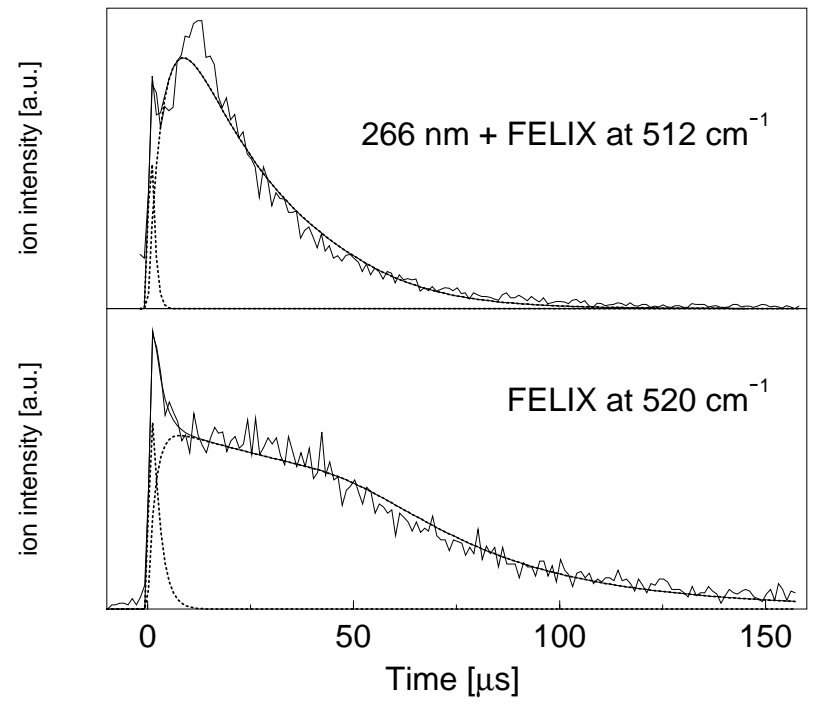

FIG. 4. $\mathrm{C}_{60}^{+}$TOB distributions obtained when exciting triplet $\mathrm{C}_{60}$ with FELIX at a frequency of $512 \mathrm{~cm}^{-1}$ (upper part) and when exciting singlet $\mathrm{C}_{60}$ with FELIX at a frequency of $520 \mathrm{~cm}^{-1}$ (lower part). The smooth curves result from fits, each being the sum of two components with the functional form as given in Eq. (2). The dotted curves give the individual components.

curves in Fig. 4 can be fit by two components of the functional form as given in Eq. (2), multiplied with the experimental detection efficiency curve (Fig. 1). In both cases, the fast component is found to decay on a time scale of a few microseconds and found to contribute less than $3 \%$ to the total signal. Comparing the two curves in Fig. 4, it is evident that the slow components are significantly different, which leads us to conclude that the slow components in both curves result from autoionization of highly vibrationally excited $\mathrm{C}_{60}$ that is either electronically excited (upper curve) or not (lower curve). It is seen that the TOB distribution originating from the triplet state surface shows a distinct peak and decays almost an order of magnitude faster than the TOB distribution originating from the ground state surface of $\mathrm{C}_{60}$. This indicates that electronic excitation to intermediate states is important in the autoionization process and that preexcited triplet $\mathrm{C}_{60}$ has already taken the first, and perhaps highest hurdle towards the ionizing state. However, it should be noted that this interpretation is in apparent contradiction to lifetimes of the triplet state of $\mathrm{C}_{60}$ [25] that could extrapolate to a very short lifetime, and therefore fast thermalization rates, at high internal energies.

A remaining question is the nature of the fast component, which becomes the dominant channel at high excitation fluences (see Fig. 2). At this point we can only speculate about the nature of this channel. One possibility is that it results from direct IR (multiphoton) excitation of $\mathrm{C}_{60}$ to an electronically excited state; at low IR pulse energies this channel resembles the TOB distribution of triplet state excited molecules, and the observed dominance of 
this channel at high IR pulse energies is as expected for a multiphoton process.

The data presented here show that the autoionization process of $\mathrm{C}_{60}$ is more complicated than previously thought. The observations are not consistent with the picture of a direct coupling of highly vibrationally excited ground state $\mathrm{C}_{60}$ to the ion. In such a scenario at least $7.6 \mathrm{eV}$ of vibrational energy, corresponding to a minimum of forty vibrational quanta, has to be exchanged to electronic energy in a single step, and the molecule appears to prefer a different route. It is observed that states that are intermediate between the vibrationally excited ground state levels and the electronic states of the ion play a crucial role in the thermal ionization process. Vibrationally and electronically excited $\mathrm{C}_{60}$ is seen to undergo autoionization on a much faster time scale than $\mathrm{C}_{60}$ that is only vibrationally excited. For $\mathrm{C}_{60}$, the first electronically excited state is a triplet state at $1.5 \mathrm{eV}$. The first excited singlet state is around $2.0 \mathrm{eV}$ above the ground state [26]. From there on, the next higher singlet and triplet states follow in close succession, and the exchange of vibrational energy with electronic energy can occur efficiently, by exchange of only one or two quanta of vibrational energy, in a stepwise manner. A bottleneck in phase space will be the transition from the electronic ground state to the first electronically excited state. Such a mechanism is consistent with the observation that molecules with lower lying electronically excited states, such as $\mathrm{C}_{70}$ and higher fullerenes, show more efficient IR laser induced autoionization [20,27].

In previous measurements on autoionization using UV/VIS lasers, only monotonically decaying time profiles have been observed. When using only the UV laser, at increased laser fluence, such time profiles are obtained in our experimental setup as well. In these experiments the initial excitation is to electronically excited states, which are assumed to decay fast to the ground electronic surface. The results presented here show that either the autoionization occurs in those experiments from electronically excited states that have not yet relaxed to the ground state surface or that the internal energy in those experiments is substantially higher, resulting in increased rate constants along the way to autoionization.

We acknowledge the support by the "Stichting voor Fundamenteel Onderzoek der Materie (FOM)" in providing the required beam time on FELIX and highly appreciate the skillful assistance by the FELIX staff. This work is part of the research program of the FOM, which is financially supported by the "Nederlandse Organisatie voor Wetenschappelijk Onderzoek (NWO)," and receives direct support by the NWO through PIONIER Grant No. 030-66-89.
[1] T. Leisner, K. Athanassenas, O. Echt, O. Kandler, D. Kreisle, and E. Recknagel, Z. Phys. D 20, 127 (1991).

[2] A. Amrein, R. Simpson, and P. Hackett, J. Chem. Phys. 94, 4663 (1991).

[3] T. Leisner, K. Athanassenas, D. Kreisle, E. Recknagel, and O. Echt, J. Chem. Phys. 99, 9670 (1993).

[4] E. E. B. Campbell, G. Ulmer, and I. V. Hertel, Phys. Rev. Lett. 67, 1986 (1991).

[5] P. Wurz and K. R. Lykke, J. Chem. Phys. 95, 7008 (1991).

[6] K. R. Lykke and P. Wurz, J. Phys. Chem. 96, 3191 (1992).

[7] P. Wurz and K. R. Lykke, J. Phys. Chem. 96, 10129 (1992).

[8] Y. Zang and M. Stuke, Phys. Rev. Lett. 70, 3231 (1993); K. R. Lykke, ibid. 75, 1234 (1995); Y. Zang and M. Stuke, ibid. 75, 1235 (1995); C. E. Klots and R. N. Compton, ibid. 76, 4092 (1996); M. Stuke and Y. Zang, ibid. 76, 4093 (1996).

[9] D. Ding, R. N. Compton, R.E. Haufler, and C. E. Klots, J. Phys. Chem. 97, 2500 (1993).

[10] D. Ding, J. Huang, R. N. Compton, C. E. Klots, and R. E. Haufler, Phys. Rev. Lett. 73, 1084 (1994).

[11] P. Wurz and K. R. Lykke, Chem. Phys. 184, 335 (1994).

[12] R. Deng and O. Echt, J. Phys. Chem. A 102, 2533 (1998).

[13] C. E. Klots, Z. Phys. D 20, 105 (1991).

[14] C. E. Klots, Chem. Phys. Lett. 186, 73 (1991).

[15] C. E. Klots, J. Chem. Phys. 100, 1035 (1994).

[16] C.E. Klots and R. N. Compton, Surf. Rev. Lett. 3, 535 (1996).

[17] K. Hansen and O. Echt, Phys. Rev. Lett. 78, 2337 (1997).

[18] V. N. Bagratashvili, M. V. Kuz'min, V.S. Letokhov, and A. N. Shibanov, JETP Lett. 37, 112 (1983).

[19] M. Hippler, M. Quack, R. Schwarz, G. Seyfang, S. Matt, and T. Märk, Chem. Phys. Lett. 278, 111 (1997).

[20] G. von Helden, I. Holleman, G. M. H. Knippels, A. F. G. van der Meer, and G. Meijer, Phys. Rev. Lett. 79, 5234 (1997).

[21] D. Oepts, A. F. G. van der Meer, and P. W. van Amersfoort, Infrared Phys. Technol. 36, 297 (1995).

[22] G. M. H. Knippels, R.F.X. A. M. Mols, A.F. G. van der Meer, D. Oepts, and P.W. van Amersfoort, Phys. Rev. Lett. 75, 1755 (1995).

[23] J. W. Arbogast, A. P. Darmanyan, C. S. Foote, Y. Rubin, F. Diederich, M. M. Alvarez, S. J. Anz, and R. L. Whetten, J. Phys. Chem. 95, 11 (1991).

[24] R. E. Haufler, L.-S. Wang, L.P.F. Chibante, C. Jin, J. Conceicao, Y. Chai, and R. E. Smalley, Chem. Phys. Lett. 179, 449 (1991).

[25] H. T. Etheridge III, R. D. Averitt, N. J. Halas, and R. B. Weisman, J. Phys. Chem. 99, 11306 (1995).

[26] S. Leach, M. Vervloet, A. Desprès, E. Bréheret, J. P. Hare, T. J. Dennis, H. W. Kroto, R. Taylor, and D. R. M. Walton, Chem. Phys. 160, 451 (1992).

[27] K. W. Kennedy and O. Echt, J. Phys. Chem. 97, 7088 (1993). 